05,11

\title{
Гомогенизационный отжиг и магнитные свойства образца фазы Лавеса $\mathrm{GdNi}_{2}$
}

\author{
(c) А.В. Маширов ${ }^{1}$, И.И. Мусабиров ${ }^{2}$, М.С. Аникин ${ }^{3}$, М.А. Сёмкин ${ }^{3}$, В. Митюк ${ }^{4}$, \\ Р.Ю. Гайфуллин ${ }^{5}$, В.В. Коледов ${ }^{1}$, В.Г. Шавров ${ }^{1}$ \\ ${ }^{1}$ Институт радиотехники и электроники им. В.А. Котельникова РАН, \\ Москва, Россия \\ ${ }^{2}$ Институт проблем сверхпластичности металлов РАН, \\ Уфа, Россия \\ ${ }^{3}$ Уральский федеральный университет, \\ Екатеринбург, Россия \\ ${ }^{4}$ Научно-практический центр НАН Беларуси по материаловедению, \\ Минск, Беларусь \\ ${ }^{5}$ Башкирский государственный университет, \\ Уфра, Россия \\ E-mail: a.v.mashirov@mail.ru
}

Поступила в Редакцию 8 июля 2021 г.

В окончательной редакции 13 июля 2021 г.

Принята к публикации 16 июля 2021 г.

\begin{abstract}
Показано влияние гомогенизационного отжига на фазовый переход ферромагнетик-парамагнетик в сплаве $\mathrm{GdNi}_{2}$. В литом образце в точке Кюри наблюдается температурный гистерезис, который во внешнем магнитном поле 13.5 Т может составлять не менее 3 градусов. Данные сканирующей электронной микроскопии электрополированных образцов показали уменьшение примесной фазы GdNi и практически отсутствие температурного гистерезиса фазового перехода в отожженном образце $\mathrm{GdNi}_{2}$. Вычислено значение изотермического изменения энтропии магнитной подсистемы $\Delta S_{m}=-18.931 \mathrm{~J} /(\mathrm{kg} \cdot \mathrm{K})$ для отожженного образца сплава $\mathrm{GdNi}_{2}$ в точке Кюри $75 \mathrm{~K}$ в магнитном поле $13.5 \mathrm{~T}$.
\end{abstract}

Ключевые слова: магнитокалорический эффект, $\mathrm{GdNi}_{2}$, фазы Лавеса.

DOI: $10.21883 /$ FTT.2021.12.51655.13s

\section{1. Введение}

В сплавах $R M_{2}(R-$ редкоземельный металл, $M=\mathrm{Ni}$, Al) в криогенной области температур наблюдается фазовый переход ферромагнетик-парамагнетик и, как следствие, высокие значения магнитокалорического эффекта в области ферромагнитной точки Кюри $[1,2]$. Поэтому при создании прототипов криогенных магнитных рефрижераторов сплавы фаз Лавеса используют в качестве рабочих тел [3]. Как будет показано ниже, гомогенизационный отжиг при синтезе данных соединений может достигать одного месяца и усложнять технологию изготовления, что обуславливает цель данной работы по исследованию возможности уменышения времени термообработки.

Рассмотрим режимы термообработки образца фазы Лавеса $\mathrm{GdNi}_{2}$ и родственных сплавов. Для соединения $\mathrm{DyNi}_{2}$ по данным фазовой диаграммы Dy-Ni [4] температура конгруэнтной реакции равна значению около $950 \mathrm{~K}$. В работе [5] сплав $\mathrm{DyNi}_{2}$ во избежание окисления при термообработке помещали в танталовую фольгу, после чего отжигали в вакууме при $1173 \mathrm{~K}$ в течение 48 часов. В работе [6] упоминаются различные методы термообработки отжига от нескольких суток до месяца при температурах 723-1073 K. Стехиометрические сплавы
$\mathrm{TbNi}_{2}$ также подвергают различной термообработке. Например, в работе [7], применялось два типа термообработки отжиг при $923 \mathrm{~K}$ в течение 720 часов или при $1473 \mathrm{~K}$ в течение 15 часов, которые дали сопоставимые результаты. Также производят термообработку образца в танталовой фольге и в вакуумированной кварцевой ампуле в течение месяца при температуре $1123 \mathrm{~K}$ [2]. Вплоть до использования метода спинингования лент, без последующего отжига [8]. Известны работы, где не описана термообработка после выплавки [9] или применяется выплавка методом индукционного нагрева [10]. Ряд работ описывающих синтез сплавов $\mathrm{GdNi}_{2}$ не содержат информацию по термообработке гомогенизационным отжигом [11]. В работе [12] сплав $\mathrm{GdNi}_{2}$ после нескольких переплавок отжигают с целью гомогенизации в течение 360 часов при температуре $1073 \mathrm{~K}$. В работах [13-15] в течение 72 часов при температурах 900, 1170 и $1200 \mathrm{~K}$, соответственно, и 96 часов при $1073 \mathrm{~K}$ [16]. Сплав $\mathrm{DyAl}_{2}$ термобрабатывают в вакуумной печи в течение 100 часов при температуре около $1073 \mathrm{~K}$ [17]. С указанием на конгруэнтное плавление для образца $\mathrm{DyAl}_{2}$ в работе [18] проводили только кратковременный деформационный отжиг (около 24 часов) при температуре около $1273 \mathrm{~K}$. Так же есть 
работа, в которой не проводился гомогенизационный отжиг [19].

\section{2. Образцы и методы эксперимента}

Методом аргонно-дуговой плавки на медном водоохлаждаемом поддоне из высокочистых навесок металлов $\mathrm{Gd}$ и $\mathrm{Ni}$ с тремя переворотами и четырьмя переплавками был синтезирован литой образец $\mathrm{GdNi}_{2}$. С целью гомогенизации запаянный в кварцевую вакуумированную ампулу образец отжигался в течение 100 часов при температуре $1073 \mathrm{~K}$ с последующим естественным охлаждением в отключенной печи.

Фазовый анализ и определение параметров кристаллической структуры соединений был выполнен с помощью метода рентгеновской дифракции, с применением порошкового дифрактометра D8 Advance (Bruker) c источником излучения $\mathrm{Cu}-K \alpha, \lambda=1.5406 \AA$ А̊. Обработка рентгенограмм проводилась с помощью полнопрофильного метода Ритвельда с применением программного обеспечения FullProf Suite [20].

Исследование микроструктуры и выявление элементного состава сплава выполнено на растровом электронном микроскопе Vega 3 SBH (Tescan) с помощью детекторов регистрации обратно-рассеянных электронов и энерго-дисперсионного анализа X-Act (Oxford Instruments). Измерения выполнены при ускоряющем напряжении $20 \mathrm{kV}$. Шлиф для исследований был приготовлен полировкой на абразивной бумаге различной зернистости и финишной полировке на алмазной суспензии.

Измерения намагниченности в магнитных полях до $13.5 \mathrm{~T}$ выполнены на универсальной установке для измерения физических свойств фирмы Cryogenic Ltd. Масса образцов составляла 151 для литого и $48 \mathrm{mg}$ ля отожженного. Скорость нагрева и охлаждения при измерении намагниченности $1-1.5 \mathrm{~K} / \mathrm{min}$.

\section{3. Результаты и обсуждение}

На рис. 1 приведены рентгенограммы образца после литья $(a)$ и отожженного в течение 100 часов при $1073 \mathrm{~K}(b)$ образцов $\mathrm{GdNi}_{2}$, отметим, что экспериментальные точки хорошо описываются расчетными линиями. Все рефлексы рентгенограмм $\mathrm{GdNi}_{2}$ хорошо описываются кубической $F d-3 m$ пространственной группой. Общий фактор расходимости рентгенограмм образцов $\chi^{2}=1.3$ и $1.2 \%$, соответственно. В кристаллической структуре $\mathrm{GdNi}_{2}$ ионы $\mathrm{Gd}$ занимают позицию $8 b$ с координатами $(3 / 8,3 / 8,3 / 8)$, ионы $\mathrm{Ni}^{+}$занимают позицию $16 c$ с координатами $(0,0,0)$. Уточнные значения параметров кристаллической решетки составляют: $a=7.2106(5) \AA$ для литого и $a=7.2131(3) \AA$ для отожженного образцов, что хорошо согласуется с литературными данными $a=7.206 \AA[11]$. Видно, что после отжига объем элементарной ячейки $\mathrm{GdNi}_{2}$ увеличился незначительно, примерно на $0.1 \%$.

Были проведены микроструктурные исследования сплава $\mathrm{GdNi}_{2}$ в состояниях после литья и гомогенизационного отжига. На рис. 2, $a$ представлены результаты исследования микроструктуры сплава в режиме регистрации обратно-рассеянных электронов (ОРЭ) в состоянии после литья. В микроструктуре наблюдается три фазы, отличных по структуре. Анализ элементного состава методом энерго-дисперсионного анализа показывает, что основная матрица соответствует интерметаллидной фазе $\mathrm{GdNi}_{2}$. Общий анализ рисунка позволяет выявить зеренный контраст основной матрицы сплава. Равноосные зерна имеют размер порядка $100 \mu \mathrm{m}$. В микроструктуре так же наблюдаются еще две фазы, имеющие светлый оттенок. Одна фаза в виде вытянутых светлых полос толщиной около $2 \mu \mathrm{m}$ и длиной до $50 \mu \mathrm{m}$. Она соответствует оксидной фазе $\mathrm{GdO}(35-65 \%)$. Наличие кислорода по всей видимости обусловлено качеством материала навески. Для его выведения необходима дополнительная термическая обработка перед внесением в навеску сплава. Судя по объемной доле содержание данной фазы относительно небольшое и не должно сказаться на интегральных функциональных свойствах исследуемого сплава. Третья фаза, имеющая сетчатую структуру, соответствует интерметаллидной фазе GdNi. Исследование микроструктуры сплава $\mathrm{GdNi}_{2}$ после гомогенизационного отжига в течение 100 часов при $1073 \mathrm{~K}$ представлено на рис. 2, $b$. Серия измерений выполнена аналогично сплаву в литом состоянии. Общий анализ микроструктуры в режиме ОРЭ показывает, что в структуре наблюдаются также три различные фазы. Однако по сравнению с исходным состоянием после гомогенизационного отжига доля матричной фазы значительно выросла. Это заметно по снижению фаз, имеющих светлые оттенки. Значительно уменьшилась доля фазы, соответствующая интерметаллидной фазе GdNi.

Таким образом, исследование микроструктуры сплава $\mathrm{GdNi}_{2}$ в литом состоянии и после гомогенизационного отжига показывают, что в исходном состоянии наблюдается значительная доля интерметаллидной фазы GdNi. В результате отжига происходит частичное растворение данной фазы и увеличение доля искомой интерметаллидной фазы $\mathrm{GdNi}_{2}$. Наличие кислорода в сплаве возможно обусловлено качеством гадолиния, использованного для навески. Для снижения доли оксидной фазы, необходима предварительная переплавка Gd для навески сплава.

Результаты измерения намагниченности образцов в зависимости от температуры при постоянном внешнем магнитном поле $0.01,1,10,13.5 \mathrm{~T}$ представлены на рис. 3 и 4 . Намагниченность при $5 \mathrm{~K}$ в магнитнном поле 100 Ое для литого и отожженного образца равна 3.1 и $3.5 \mathrm{emu} / \mathrm{g}$, соответственно, а в магнитном поле $135 \mathrm{kOe}$ около $139 \mathrm{emu} / \mathrm{g}$ для обоих образцов. На данных рисунках видно, что при внешнем магнитном поле в 

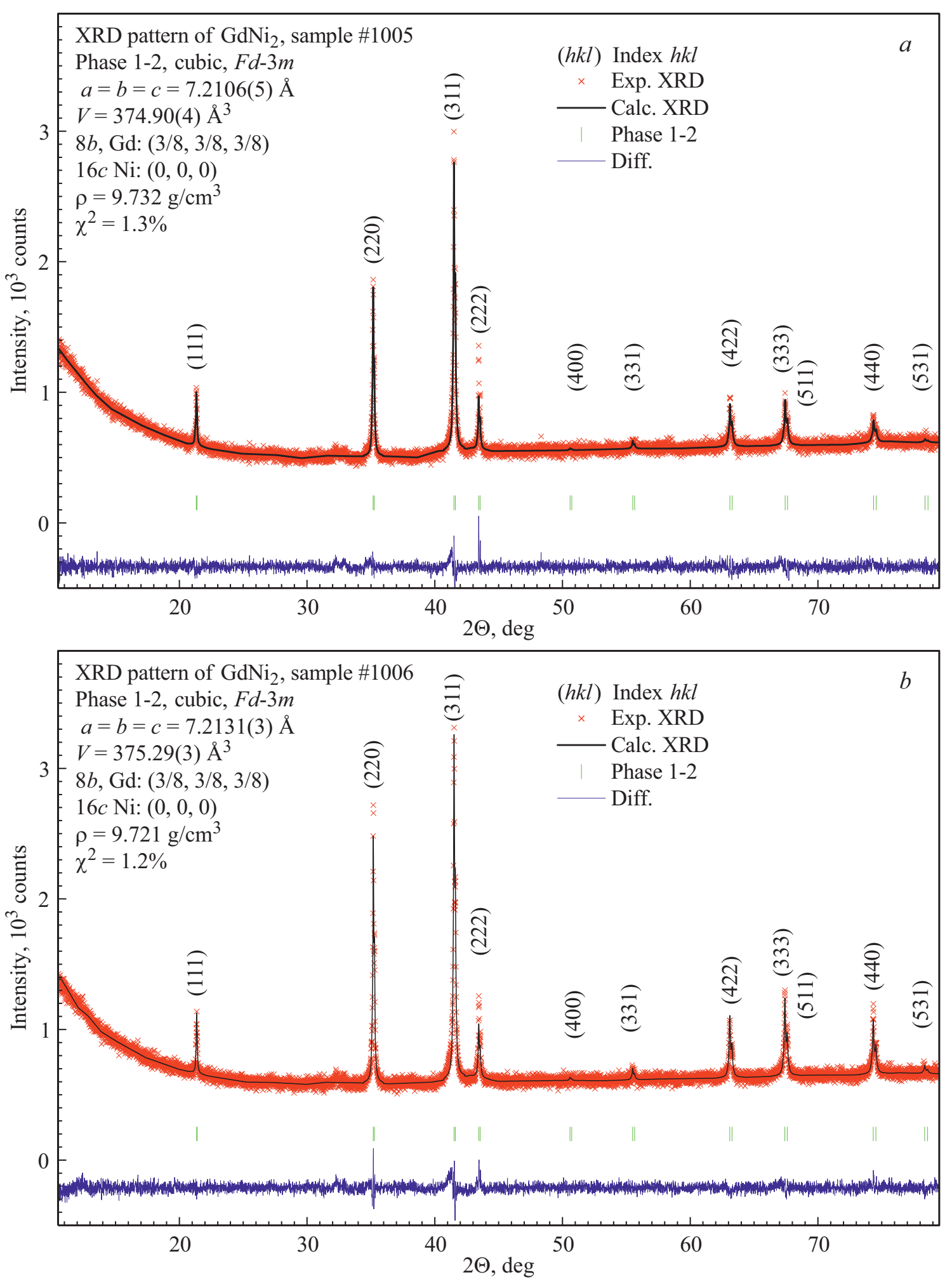

Рис. 1. Экспериментальные (точки) и расчетные (линия) рентгенограммы образцов $\mathrm{GdNi}_{2}$ после литья $(a)$ и отожженного в течение 100 часов при $1073 \mathrm{~K}(b)$; линии внизу — разность между рентгенограммами; ряд штрихов - угловые положения Брэгговских рефлексов основной фазы, в круглых скобках приведены соответствующие индексы Миллера.

точке Кюри равной $75 \mathrm{~K}$ для образца после литья наблюдается более широкий температурный гистерезис, чем у гомогенизированного, согласно таблице. Наличие температурного гистерезиса около 1 градуса у гомогенизированного образца можно объяснить условиями эксперимента. Литой образец демонстрирует увеличение 

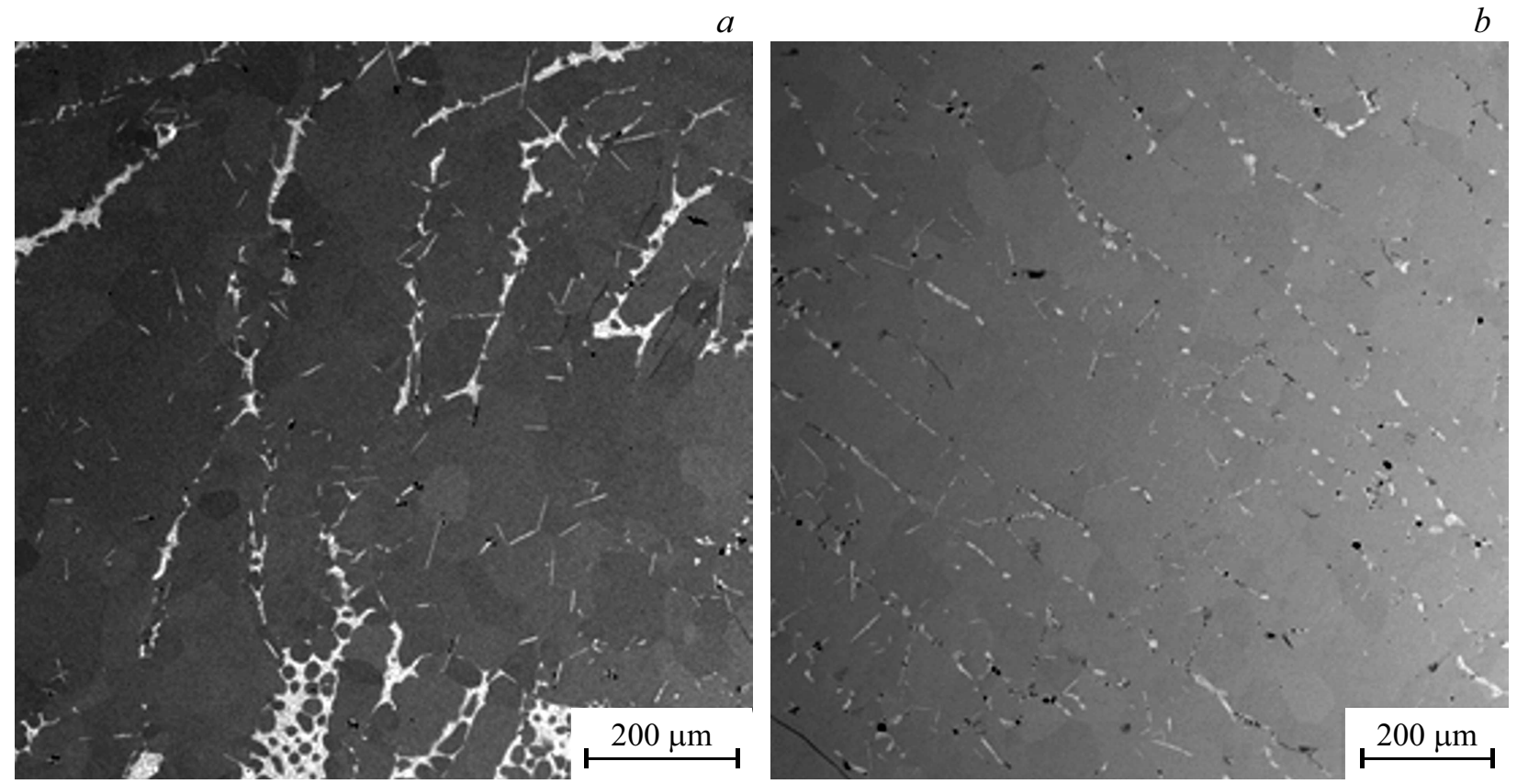

Рис. 2. Микроструктура сплава $\mathrm{GdNi}_{2}$ в состоянии после литья $(a)$ и после гомогенизационного отжига в течение 100 часов при $1073 \mathrm{~K}(b)$.

Значения температурного гистерезиса в точке Кюри

\begin{tabular}{l|c|c|c|c|c}
\hline \multicolumn{1}{c|}{ Магнитное поле, Т } & 0.01 & 0.5 & 1 & 9 & 13.5 \\
\hline $\begin{array}{l}\text { Температурный гистерезис } \\
\text { образца после литья, K }\end{array}$ & 2.06 & 2.44 & 2.44 & 4.07 & 3.66 \\
\hline $\begin{array}{l}\text { Температурный гистерезис } \\
\text { отожженного образца, K }\end{array}$ & 1.34 & 1.03 & 1.03 & 0.23 & 0.23
\end{tabular}

температурного гистерезиса до значений около 4 градусов. По-видимому это можно объяснить наличием значительной доли интерметаллидной фазы GdNi, которая при отжиге растворяется с увеличением доли материнской фазы $\mathrm{GdNi}_{2}$. Известно, что температуры Кюри для интерметаллида GdNi равна значению около $80 \mathrm{~K}$ [21].

Изотермические кривые технического намагничивания в магнитном поле до $13.5 \mathrm{~T}$ при температурах от 5 до $120 \mathrm{~K}$ представлены на рис. 5. Насыщение кривой технического намагничивания наблюдается в $1.25 \mathrm{~T}$ со значением для образца после литья и отожженного 134.9 и $134.6 \mathrm{emu} / \mathrm{g}$ соответственно. Указанные значения слабо изменяются при увеличении магнитного поля до $13.5 \mathrm{~T}$ и равны 138.5 и $137.5 \mathrm{emu} / \mathrm{g}$ соответственно. Используя изотермические кривые технического намагничивания можно вычислить магнитокалорический эффект в виде изотермического изменения энтропии магнитной подсистемы $\Delta S_{m}$ [22]. На рис. 6 представлены результаты данного вычисления, которые составили максимальное значение в точке Кюри в магнитном поле 13.5 Т для литого и отожженного образцов -18.616 и $-18.931 \mathrm{~J} /(\mathrm{kg} \cdot \mathrm{K})$ соответственно. Это значение почти в два раза меньше значения $\Delta S_{m}$ для $\mathrm{GdNi}$ равное $-31.2 \mathrm{~J} /(\mathrm{kg} \cdot \mathrm{K})$ в поле $14 \mathrm{~T}$ при температуре Кюри $80 \mathrm{~K}$ [21]. В работе [11] значение $\Delta S_{m}$ магнитокалорического эффекта в точке Кюри $70 \mathrm{~K}$ для $\mathrm{GdNi}_{2}$ в магнитом поле $10 \mathrm{~T}$ составило $-17 \mathrm{~J} /(\mathrm{kg} \cdot \mathrm{K})$, против $-15.8 \mathrm{~J} /(\mathrm{kg} \cdot \mathrm{K})$ при $75 \mathrm{~K}$ в данной работе для отожженного образца. В работе [23] значение $\Delta S_{m}$ магнитокалорического эффекта в точке Кюри $75 \mathrm{~K}$ для сфер $\mathrm{GdNi}_{2}$ диаметром 500-850 $\mu \mathrm{m}$ в магнитом поле $5 \mathrm{~T}$ составило $-3.9 \mathrm{~J} /(\mathrm{kg} \cdot \mathrm{K})$, против $-9.9 \mathrm{~J} /(\mathrm{kg} \cdot \mathrm{K})$ массивных образцов в данной работе.

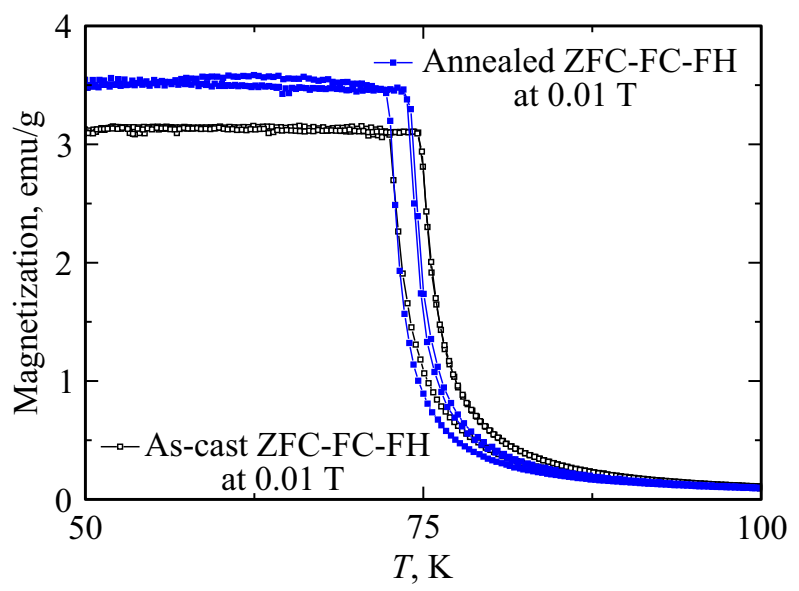

Рис. 3. Зависимость намагниченности образца после литья (черный) и отожженного (синий) от температуры в магнитном поле $0.01 \mathrm{~T}$. 
Таким образом, термообработка в виде отжига в течение 100 часов при $1073 \mathrm{~K}$ сплава $\mathrm{GdNi}_{2}$ не сказывается на значении изотермического магнитокалорического эффекта, она уменьшает ширину температурного гистерезиса, обусловленного неоднородностью образца, что важно при прямом измерении магнитокалорического эффекта в режиме нагрева или охлаждения.

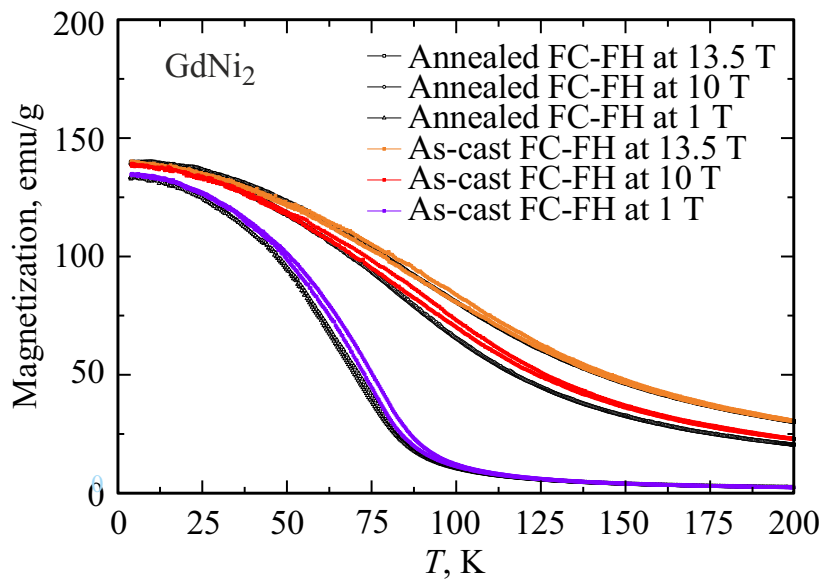

Pис. 4. Зависимость намагниченности образца после литья и отожженного от температуры в магнитных полях 1, 10, 13.5 T.
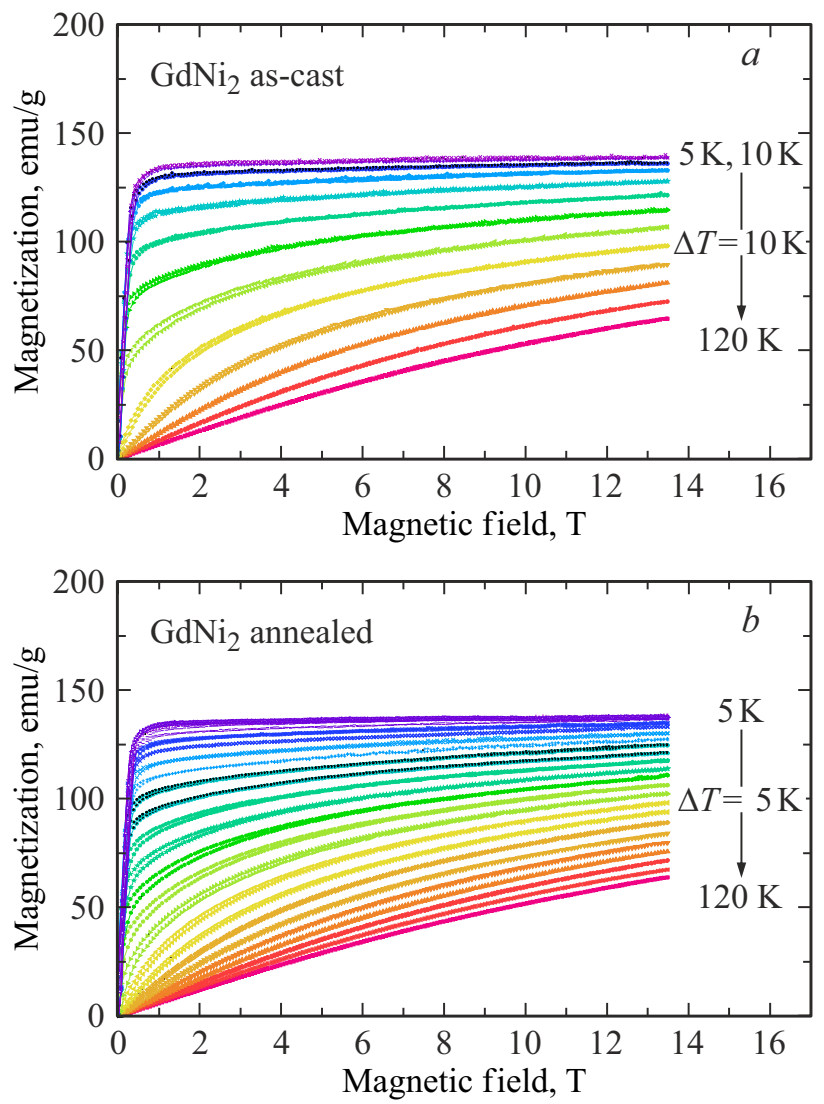

Pис. 5. Кривые изотермического технического намагничивания до 13.5 Т после литья $(a)$ и отожженного $(b)$ образца сплава $\mathrm{GdNi}_{2}$.
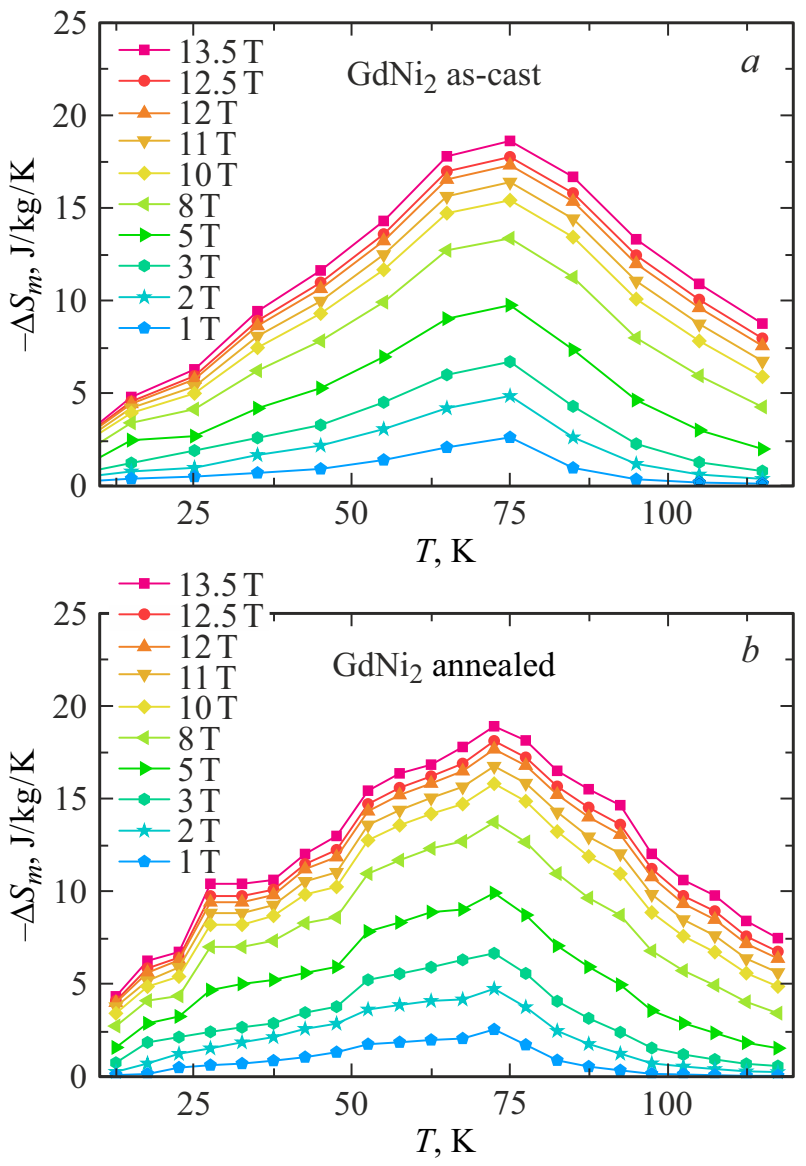

Рис. 6. Температурная зависимость изотермического изменения энтропии магнитной подсистемы после литья $(a)$ и отожженного (b) образца сплава $\mathrm{GdNi}_{2}$.

\section{4. Заключение}

Гомогенизирующий отжиг уменьшает количество примесной фазы в сплаве фазы Лавеса $\mathrm{GdNi}_{2}$. Температурный гистерезис в точке Кюри данного сплава в неотожженном состоянии может достигать значений 3 градусов. Термообработка в виде отжига в течение 100 часов при $1073 \mathrm{~K}$ уменьшает значение температурного гистерезиса до значений порядка инструментальной погрешности измерений намагниченности. Полученное значение $\Delta S_{m}$ магнитокалорического эффекта в данной работе для отожженного образца $\mathrm{GdNi}_{2}$ в точке Кюри $75 \mathrm{~K}$ в магнитном поле $13.5 \mathrm{~T}$ равно $-18.931 \mathrm{~J} /(\mathrm{kg} \cdot \mathrm{K})$, что значительно меньше значения $\Delta S_{m}$ для GdNi равное $-31.2 \mathrm{~J} /(\mathrm{kg} \cdot \mathrm{K})$ в поле 14 Т при температуре Кюри $80 \mathrm{~K}$.

\section{Финансирование работы}

Исследование выполнено за счет гранта Российского научного фонда (проект № 20-79-10197). Исследование микроструктуры с помощью растровой электронной микроскопии выполнено на базе Центра коллективного пользования научным оборудованием Института про- 
блем сверхпластичности металлов РАН „Структурные и физико-механические исследования материалов“.

\section{Конфликт интересов}

Авторы заявляют, что у них нет конфликта интересов.

\section{Список литературы}

[1] J. Cwik, Y. Koshkid'ko, K. Nenkov, E.A. Tereshina, K. Rogacki. J. Alloys Comp. 735, 1088 (2018.).

[2] J. Ćwik, Y. Koshkid'ko, N.A. de Oliveira, K. Nenkov, A. Hackemer, E. Dilmieva, N. Kolchugina, S. Nikitin, K. Rogacki. Acta Mater. 133, 230 (2017).

[3] I. Park, S. Jeong. Cryogenics 88, 106 (2017).

[4] M. Li, H. Wei. Calphad 33, 3, 517 (2009).

[5] A. Tomokiyo, H. Yayama, H. Wakabayashi, T. Kuzuhara, T. Hashimoto, M. Sahashi, K. Inomata. Specific Heat and Entropy of $\mathrm{RNi}_{2}$ (R: Rare Earth Heavy Metals) in Magnetic Field. In Advances in Cryogenic Engineering Materials Springer, Boston, MA (1986). P. 295-301.

[6] P.J. Ibarra-Gaytan, C.F. Sánchez-Valdes, J.L. Sánchez Llamazares, P. Álvarez- Alonso, P. Gorria, J.A. Blanco. Appl. Phys. Lett. 103, 15, 152401 (2013).

[7] E. Gratz, E. Goremychkin, M. Latroche, G. Hilscher, M. Rotter, H. Müller, A. Lindbaum, H. Michor, V. Paul-Boncour, T. Fernandez-Diaz. J. Phys. Condens. Matter 11, 40, 7893 (1999).

[8] J.L. Sánchez Llamazares, C.F. Sánchez-Valdes, P.J. IbarraGaytan, P. Álvarez-Alonso, P. Gorria, J.A. Blanco. J. Appl. Phys. 113, 17, 17A912 (2013).

[9] J.L. Wang, M.F. Md Din, S.J. Kennedy, F. Hong, S.J. Campbell, A.J. Studer, G.H. Wu, Z.X. Cheng, S.X. Dou. J. Appl. Phys. 115, 17, 17E135 (2014).

[10] H. Oesterreicher, J. Stanley, R. Pitts. Phys. Status Solidi A 12, 2, K65 (1972).

[11] S. Taskaev, V. Khovaylo, K. Skokov, W. Liu, E. Bykov, M. Ulyanov, D. Bataev, A. Basharova, M. Kononova, D. Plakhotskiy, M. Bogush, T. Gottschall, O. Gutfleisch. J. Appl. Phys. 127, 233906 (2020).

[12] S.K. Malik, W.E. Wallace. Solid State Commun. 24, 417 (1977).

[13] M. Mizumaki, K. Yano, I. Umehara, F. Ishikawa, K. Sato, A. Koizumi, N. Sakai, T. Muro. Phys. Rev. B 67, 132404 (2003).

[14] K. Yano, Y. Tanaka, I. Matsumoto, I. Umehara, K. Sato, H. Adachi, H. Kawata. J. Phys.: Condens. Matter 18, 6891 (2006).

[15] K. Yano, I. Umehara, T. Miyazawa, Y. Adachi, K. Sato. Physica B Condens. Matter 367, 81 (2005).

[16] J.A. Cannon, J.I. Budnick, R.S. Craig, S.G. Sankar, D.A. Keller. AIP Conf. Proc. 10, 1, 905 (1973).

[17] N. Nereson, C. Olsen, G. Arnold. J. Appl. Phys. 37, 12, 4575 (1966).

[18] T. Inoue, S.G. Sankar, R.S. Craig, W.E. Wallace, K.A. Gschneider Jr. J. Phys. Chem. Solids 38, 487 (1977).
[19] S. Taskaev, V. Khovaylo, K. Skokov, W. Liu, E. Bykov, M. Ulyanov, D. Bataev, A. Basharova, M. Kononova, D. Plakhotskiy, M. Bogush, M. Gavrilova, T. Gottschall, Z. Hu. Chelyabinsk Phys. Mathem. J. 5, 4 (2), 618 (2020).

[20] J. Rodríguez-Carvajal. Physica B Condens. Matter 192, 1-2, 55 (1993).

[21] R. Rajivgandhi, J. Arout Chelvane, S. Quezado, S.K. Malik, R. Nirmala. J. Magn. Magn. Mater. 433, 169 (2017).

[22] V.K. Pecharsky, K.A. Gschneidner Jr. J. Appl. Phys. 86, 1, 565 (1999).

[23] K. Matsumoto, K. Asamato, Y. Nishimura, Y. Zhu, S. Abe, T. Numazawa. J. Phys.: Conf. Ser. 400, 052020 (2012).

Редактор Д.В. Жуманов 